\title{
Effective Spring Stiffness for a Planar Periodic Array of Collinear Cracks at an Interface between Two Dissimilar Isotropic Materials
}

\author{
Huseyin Lekesiz, \\ Dept. of Mechanical Engineering, The Ohio State University, Columbus OH 43210 \\ Noriko Katsube*, \\ Dept. of Mechanical Engineering, The Ohio State University, Columbus $\mathrm{OH} 43210$ \\ Stanislav I. Rokhlin, and \\ Dept. of Materials Science, The Ohio State University, Columbus OH 43210 \\ Robert R. Seghi \\ College of Dentistry, The Ohio State University, Columbus OH 43210
}

\begin{abstract}
Explicit analytical expressions are obtained for the longitudinal and transverse effective spring stiffnesses of a planar periodic array of collinear cracks at the interface between two dissimilar isotropic materials; they are shown to be identical in a general case of elastic dissimilarity (the well-known open interface crack model is employed for the solution). Since the interfacial spring stiffness can be experimentally determined from ultrasound reflection and transmission analysis, the proposed expressions can be useful in estimating the percentage of disbond area between two dissimilar materials, which is directly related to the residual strength of the interface. The effects of elastic dissimilarity, crack density and crack interaction on the effective spring stiffness are clearly represented in the solution. It is shown that in general the crack interaction weakly depends on material dissimilarity and, for most practical cases, the crack interaction is nearly the same as that for crack arrays between identical solids. This allows approximate factorization of the effective spring stiffness for an array of cracks between dissimilar materials in terms of an elastic dissimilarity factor and two factors obtained for cracks in a homogeneous material: the effective spring stiffness for non-interacting (independent) cracks and the crack interaction factor. In order to avoid the effect of the crack surface interpenetration zones on the effective spring stiffness, the range of the tensile to transverse load ratios is obtained under the assumption of small-scale contact conditions. Since real cracks are often slightly open (due to prior loading history and plastic deformation), it is demonstrated that for ultrasound applications the results obtained are valid for most practical cases of small interfacial cracks as long as the mid-crack opening normalized by the crack length is at least in the order of $10^{-5}$.
\end{abstract}

\section{Keywords}

crack interaction; dissimilar materials; interface; spring stiffness; ultrasound

\footnotetext{
(C) 2010 Elsevier Ltd. All rights reserved.

"corresponding author: katsube.1@osu.edu, Phone: (614) 292-0971, Fax: (614) 292-3163.
}

Publisher's Disclaimer: This is a PDF file of an unedited manuscript that has been accepted for publication. As a service to our customers we are providing this early version of the manuscript. The manuscript will undergo copyediting, typesetting, and review of the resulting proof before it is published in its final citable form. Please note that during the production process errors may be discovered which could affect the content, and all legal disclaimers that apply to the journal pertain. 


\section{INTRODUCTION}

Layered materials are extensively used in various products and devices to improve structural performance such as strength and durability. Thermal barrier coatings, for example, are used in turbine blades to protect the core material from thermal fatigue (Miller, 1987). In the field of dentistry, resin-retained ceramic restorations are performed to protect remaining teeth and restore mechanical function without loss of aesthetics (Wang et al., 2007). Wide applications of adhesive bonds in aerospace industry for both aluminum and composite structures are well documented. Failures of these layered structures are often attributed to interfacial damage in the form of micro-cracks or debonded zones, which are either preexisting or developed during service due to mechanical/thermal fatigue and environmental degradations.

Ultrasonic methods have been widely used to detect and characterize interfacial imperfections such as distributed micro-cracks or micro-disbonds (Thompson and Thompson, 1991; Rokhlin et al., 2004). For modeling of ultrasonic wave interactions at imperfect interfaces, a quasi-static approximation (Baik and Thompson, 1984; Margetan et al., 1988) has been widely used. In this model the reduction in static stiffness of the overall structure due to compromised interfaces (micro-cracks or micro-disbonds) is represented by continuous, uniform distributions of interfacial springs. It has been demonstrated by Angel and Achenbach (1985) that the quasi-static approximation is applicable at low frequencies, when the size of the imperfections is much smaller than the wavelength.

Significant experimental and theoretical advances have been made towards inversion of the interfacial stiffness distributions from ultrasonic measurements (Wang and Rokhlin, 1991; Rokhlin et al., 2004; Baltazar et al., 2003; Wang et al., 2006; Leiderman et al., 2007) and relating the spring stiffness constants to the micromechanical and geometric properties of the micro-cracks (Baik and Thompson, 1984; Lavrentyev and Rokhlin, 1994; Pecorari and Kelly, 2000). Explicit expressions of effective spring stiffness in terms of the crack geometry and density are important since they can be used to estimate the percentage of disbond area (Palmer et al., 1988), which is critical in assessing the bond integrity and the remaining life.

Using an available fracture mechanical model, Baik and Thompson (1984) have obtained the expression for effective normal spring stiffness for a planar array of periodically spaced strip cracks in a homogeneous material. For this geometry, Angel and Achenbach (1985) have obtained an exact solution of the elastodynamic reflection/refraction boundary-value problem and have numerically compared the exact solution with the quasi-static (spring) approximation. They have demonstrated that for this problem the approximation is suitable for $a / b<0.8\left(a / b\right.$ : crack density, see Figure 1 (a)) at $2 b / \lambda_{T}<0.25\left(\lambda_{T}\right.$ is a transverse wavelength). The applicability range of the $2 b / \lambda_{T}$ ratio decreases with increase of crack density $a / b$ (increase of crack interaction). Excellent agreement between the spring approximation and experiment has been obtained by Palmer et al. (1988) who have reported measurements of the ultrasonic reflectivity on imperfectly diffusion-bonded samples.

For non-interacting cracks at the interface between two dissimilar materials, Pecorari and Kelly (2000) have obtained an explicit expression of the effective normal spring stiffness and have shown that their expression reduces to that of Baik and Thompson (1984) when elastic moduli difference and crack area fraction approach zero. While crack interactions have been investigated in regard to overall effective material properties (Nemat-Nasser et al., 1993), the analysis of effective spring stiffness for interacting cracks between dissimilar materials is not available. 
In this work, for a planar periodic array of collinear cracks between two dissimilar isotropic materials (Figure 1 (a)), explicit expressions for effective transverse and normal spring stiffnesses have been obtained; they are shown to be identical in a general case of elastic dissimilarity. We have examined the effect of an elastic dissimilarity and crack interaction on the effective interfacial spring stiffness (Figure 1 (b)). Our derivation is based on the elastic analysis of a periodic array of cracks between two dissimilar materials under normal and transverse loading in the framework of the open crack model (Williams, 1959; England, 1965; Erdogan, 1965; Rice, 1988; Hutchinson and Suo, 1992). By inserting the stress intensity factor (Rice and Sih, 1965; Sih, 1973) into the expression for the strain energy release rate (Hutchinson and Suo, 1992) and using Castigliano's theorem extended for cracked bodies (Tada et al., 2000), the effective spring stiffness for cracks between dissimilar materials is obtained.

The effect of elastic dissimilarity, crack density and crack interaction on the effective spring stiffness is explicitly expressed, and it is demonstrated that the effect of elastic dissimilarity on the crack interaction is insignificant and the crack interaction is nearly the same as that for crack arrays between identical solids. This allows approximate factorization of the effective spring stiffness for an array of cracks between dissimilar materials in terms of the elastic dissimilarity factor (elastic mismatch), the crack interaction factor, and the effective spring stiffness for non-interacting (independent) cracks in a homogeneous material as shown in Figure 1 (c). Both the theoretical limitations related to the open crack assumption and the practical usability of the obtained results in ultrasonic method applications are discussed.

\section{STRAIN ENERGY RELEASE RATE FOR AN ARRAY OF INTERFACIAL CRACKS BETWEEN TWO DISSIMILAR MATERIALS}

\subsection{The Strain Energy Release Rate Expressed in terms of $\boldsymbol{\sigma}$ and $\mathbf{T}$}

Consider an infinite array of equal cracks of length $2 a$ spaced at a constant interval $2 b$ along the bond line of two dissimilar isotropic materials with Young's moduli of $E_{1}, E_{2}$ and Poisson's ratios of $v_{1}$ and $v_{2}$. As shown in Figure 1 (a), uniform normal $(\sigma)$ and shear $(\tau)$ stresses are applied at infinity. The complex stress intensity factor, $K$, is obtained by Rice and $\operatorname{Sih}(1965)$ as

$$
K=K_{I}+i K_{I I}=\sqrt{\pi} \cosh (\pi \varepsilon)\left(k_{1}+i k_{2}\right)
$$

where parameters $k_{1}, k_{2}$ are given in the Appendix and $\varepsilon$ is called the oscillation index that can be expressed in terms of the second Dundurs' parameter $\beta$ [Dundurs (1967); Dundurs and Bogy (1969)]:

$$
\begin{gathered}
\varepsilon=\frac{1}{2 \pi} \log \left(\frac{1+\beta}{1-\beta}\right), \quad(2) \\
\beta=\frac{G_{2}\left(\kappa_{1}-1\right)-G_{1}\left(\kappa_{2}-1\right)}{G_{2}\left(\kappa_{1}+1\right)+G_{1}\left(\kappa_{2}+1\right)},
\end{gathered}
$$

where $G_{1}$ and $G_{2}$ are the shear moduli of materials 1 and 2, and

$$
\kappa_{i}=3-4 v_{i}, \quad i=1,2 \quad \text { for plane strain, }
$$




$$
\kappa_{i}=\left(3-v_{i}\right) /\left(1+v_{i}\right), \quad i=1,2 \quad \text { for plane stress. }
$$

We use the strain energy release rate (SERR) in a general form given by Hutchinson and Suo (1992) (Eq. (2.29))

$$
W=\frac{1}{E^{*} \cosh ^{2}(\pi \varepsilon)} K \bar{K}, \quad(6)
$$

where $E^{*}$ is

$$
\frac{1}{E^{*}}=\frac{1}{2}\left(\frac{1}{\overline{E_{1}}}+\frac{1}{\overline{E_{2}}}\right)=\frac{\left(1+\kappa_{1}\right)}{8(1+\alpha) G_{1}},
$$

and

$$
\begin{gathered}
\overline{E_{i}}=\frac{E_{i}}{1-v_{i}{ }^{2}} \text { for plane strain } \\
\overline{E_{i}}=E_{i} \text { for plane stress }(i=1,2)
\end{gathered}
$$

The parameter a in Eq. (7) is the first Dundurs' parameter [Dundurs (1967); Dundurs and Bogy (1969)], which is expressed in the form:

$$
\alpha=\frac{G_{2}\left(\kappa_{1}+1\right)-G_{1}\left(\kappa_{2}+1\right)}{G_{2}\left(\kappa_{1}+1\right)+G_{1}\left(\kappa_{2}+1\right)}=\frac{\overline{E_{2}}-\overline{E_{1}}}{\overline{E_{2}}+\overline{E_{1}}},
$$

Thus both Dundurs' parameters $a$ and $\beta$ characterize the elastic mismatch between two semispaces.

By inserting Eqs. (1, A2, A3) into Eq. (6), the SERR can be expressed in terms of $\sigma$ and $\tau$ as follows:

$$
W_{\text {dissimilar }}^{\text {array }}=\frac{1}{4} \frac{\left(1+\kappa_{1}\right)\left(1-\beta^{2}\right)}{G_{1}(1+\alpha)} \pi a\left\{\frac{\cosh ^{2}\left(\frac{\pi a \varepsilon}{b}\right)-\cos ^{2}\left(\frac{\pi a}{2 b}\right)}{\left(\frac{\pi a}{2 b}\right) \sin \left(\frac{\pi a}{b}\right)}\right\}\left(\sigma^{2}+\tau^{2}\right)
$$

The subscript 'dissimilar' indicates that the two materials are different, and the superscript 'array' indicates that the cracks are periodically aligned. By using Taylor series expansions and letting $a / b$ approach zero, Eq. (11) reduces to the SERR for non-interacting (independent) interfacial cracks.

$$
W_{\text {dissimilar }}^{\text {non-interacting }}=\frac{1}{8} \frac{\left(1+\kappa_{1}\right)\left(1-\beta^{2}\right)}{G_{1}(1+\alpha)} \pi a\left(1+4 \varepsilon^{2}\right)\left(\sigma^{2}+\tau^{2}\right)
$$

The same expression can be obtained directly by using the SIFs for non-interacting cracks given in Rice and Sih (1965).

By setting $a=\beta=\varepsilon=0$, Eq. (11) reduces to the SERR for an array of cracks in a homogenous material. 


$$
W_{\text {homogeneous }}^{\text {array }}=\frac{1}{8} \frac{\left(1+\kappa_{1}\right)}{G_{1}} \pi a\left\{\frac{\tan \left(\frac{\pi a}{2 b}\right)}{\left(\frac{\pi a}{2 b}\right)}\right\}\left(\sigma^{2}+\tau^{2}\right)
$$

Similarly, Eq. (12) reduces to the SERR for a non-interacting crack in a homogenous material.

$$
W_{\text {homogeneous }}^{\text {non-interacting }}=\frac{1}{8} \frac{\left(1+\kappa_{1}\right)}{G_{1}} \pi a\left(\sigma^{2}+\tau^{2}\right)
$$

\subsection{Effect of Material Dissimilarity and Crack Interaction on the SERR}

It is advantageous to represent the SERR equation (11) with use of Eqs. (12) and (14) as

$$
W_{\text {dissimilar }}^{\text {array }}=I_{W} \times M_{W} \times W_{\text {homogeneous }}^{\text {non-interacting }},
$$

where

$$
I_{W}\left(\frac{a}{b}, \varepsilon\right)=\frac{W_{\text {dissimilar }}^{\text {array }}}{W_{\text {dissimilar }}^{\text {non-interacting }}}=\frac{\cosh ^{2}\left(\frac{\pi a \varepsilon}{b}\right)-\cos ^{2}\left(\frac{\pi a}{2 b}\right)}{\left(\frac{\pi a}{2 b}\right) \sin \left(\frac{\pi a}{b}\right)} \frac{2}{\left(1+4 \varepsilon^{2}\right)},
$$

and

$$
M_{W}(\alpha, \beta)=\frac{W_{\text {dissimilar }}^{\text {non-interacting }}}{W_{\text {hom-interacting }}^{\text {noneneous }}}=\frac{\left(1-\beta^{2}\right)\left(1+4 \varepsilon^{2}\right)}{(1+\alpha)}
$$

It is important to note that $I_{W}$, defined by Eq. (16), describes the effect of crack interaction on the SERR and depends on both the oscillation index $\varepsilon$, which is a function of the elastic properties of both semispaces, and the crack density a/ $b$. The factor $M_{W}$ (Eq. (17)) depends solely on the dissimilarity of the elastic properties of both semispaces, and it represents the effect of material dissimilarity on the SERR. It is also important to note that for an arbitrary bi-material combination $|\beta|<0.5$ and $|\varepsilon|<\frac{1}{2 \pi} \ln (3) \approx 0.1748$, where the limits are reached in the extreme cases when the shear modulus ratio $G_{2} / G_{1}$ and the Poisson's ratio $\mathrm{v}_{2}$ (or $G_{1} / G_{2}$ and $v_{1}$ ) approach zero under the plane strain condition. In the extreme case where $\beta=0.5$ and $a=1$, material factor $M_{W}$ approximately reaches the minimum value of 0.4209 , and it becomes equal to 1 for identical semispaces $(\beta=0, a=0)$. As the value of $a$ approaches -1 ( $G_{2} / G_{1}$ approaches zero), $M_{W}$ becomes unbounded. Therefore, Eq. (15) shows that the SERR for a periodic array of cracks at the interface between two dissimilar materials can be exactly factorized as a product of three components: elastic dissimilarity function, $M_{W}$, crack interaction function, $I_{W}$, and the SERR for non-interacting cracks in a homogenous material.

For most practical applications, $\varepsilon$ remains small $(|\varepsilon|<0.05)$. In this case, using Taylor series expansions, the crack interaction function, $I_{W}$, can be approximated as

$$
I_{W}\left(\frac{a}{b}, \varepsilon\right) \approx I_{W} \text { approximate }\left(\frac{a}{b}\right)=\frac{\tan \left(\frac{\pi a}{2 b}\right)}{\left(\frac{\pi a}{2 b}\right)} .
$$


Thus $I_{W}$ approximate depends only on the crack geometry and is independent of the oscillation index, $\varepsilon$ (elastic mismatch). It is worth noting here that this function is identical to the interaction function for cracks in a homogenous material defined by

$$
I_{W} \text { homogenous }\left(\frac{a}{b}\right)=\frac{W_{\text {homogeneous }}^{\text {array }}}{W_{\text {homogeneous }}^{\text {non-int }}} \frac{\tan \left(\frac{\pi a}{2 b}\right)}{\left(\frac{\pi a}{2 b}\right)} .
$$

To illustrate the accuracy of this approximation we introduce the relative error $e$ (in percent)

$$
e\left(\frac{a}{b}, \varepsilon\right)=\frac{I_{W}{ }^{\text {approximate }}\left(\frac{a}{b}\right)-I_{W}\left(\frac{a}{b}, \varepsilon\right)}{I_{W}\left(\frac{a}{b}, \varepsilon\right)} \times 100 .
$$

Figure 2 shows the error $e$ contours in coordinates of the oscillation index $\varepsilon$ and crack density $a / b$. Some practical material combinations taken from Hutchinson et al. (1987) are also indicated by horizontal dashed lines. As can be seen, the error reaches maximum as the material mismatch and crack density approach maximum values. However, for most practical applications, $e$ is less than $2.5 \%$ even for crack density $a / b$ approaching one (when spacing between the cracks is zero). Therefore, it is possible to neglect the effect of elastic dissimilarity on the crack interaction for most realistic applications and use as an interaction function that obtained for the identical semispaces.

\section{EFFECTIVE INTERFACIAL SPRING STIFFNESS}

\subsection{Effective Spring Stiffness}

In the problem considered in Figure 1(a), the far field displacement due to applied tractions can be separated into displacement components of perfectly bonded semispaces (no cracks at the interface) and an additional displacement due to the presence of the crack array

$$
\Delta=\Delta_{\text {perfect-bonding }}+\Delta_{\text {crack }}
$$

The idea is to replace the array of cracks by continuously distributed interfacial springs, such that they provide the same additional interface compliance (additional displacement $\Delta_{\text {crack }}$ ) as due to the crack array (Figure 1(b)). Following the work by Baik and Thompson (1984), the normal and tangential spring stiffness, $k_{N}$ and $k_{T}$, are defined by

$$
k_{N}=\frac{\sigma}{\Delta_{N, \text { crack }}}, k_{T}=\frac{\tau}{\Delta_{T . \text { crack }}},
$$

where $\Delta_{N, c r a c k}$ and $\Delta_{T, c r a c k}$, respectively, represent the additional displacement in the normal and transverse directions. The displacements can be calculated by using Castigliano's theorem extended to cracked bodies (Tada et al., 2000):

$$
\Delta_{\text {crack }}=\frac{\partial}{\partial Q}\left(2 \int_{0}^{a} W d x\right)
$$

where $Q$ is the applied load per unit crack length along the coordinate $z$. Eq. (23) can also be obtained without invoking Castigliano's theorem. The strain energy (due to introducing the crack) in the loaded body can be written in terms of the interfacial stiffness and the far-field tractions by considering the work done in applying these tractions. The displacement due to 
the crack can then be obtained by differentiating the strain energy with respect to the farfield load. Since the strain energy release rate is a known function, the strain energy can be obtained by integrating with respect to the crack length. By inserting Eq. (11) into Eq. (23) and use of (22), we obtain

$$
k_{N, \text { dissimilar }}^{\text {array }}=k_{T, \text { dissimilar }}^{\text {array }}=\frac{G_{1}}{b\left(1+\kappa_{1}\right)} \frac{(1+\alpha)}{\left(1-\beta^{2}\right) L\left(\frac{a}{b}, \varepsilon\right)},
$$

where

$$
L\left(\frac{a}{b}, \varepsilon\right)=\int_{0}^{\frac{a}{b}} \frac{1}{\sin (\pi \bar{x})}\left[\cosh ^{2}(\pi \bar{x} \varepsilon)-\cos ^{2}\left(\frac{\pi \bar{x}}{2}\right)\right] d \bar{x}
$$

Equality of normal and transverse spring stiffness equation (24) is a generalization of a known result for a $2 \mathrm{D}$ crack array in a homogeneous space. The result is not obvious due to coupling of normal and transverse tractions in the stress intensity factors (A1-A3).

In Eq. (25), crack density, $a / b$, and elastic mismatch represented by the oscillation index, $\varepsilon$, are coupled. In Section 2, however, we have shown that the effect of crack interaction and elastic mismatch on the SERR can be approximately decoupled when $\varepsilon$ is small (which is the case for most realistic material pairs). Motivated by the results in Section 2, the Taylor series expansion is employed in Eq. (25) for small $\varepsilon(|\varepsilon|<0.05)$ which results in the following approximation for $L\left(\frac{a}{b}, \varepsilon\right)$ :

$$
L\left(\frac{a}{b}, \varepsilon\right) \approx L^{a p p r o x i m a t e}\left(\frac{a}{b}, \varepsilon\right)=\frac{\left(1+4 \varepsilon^{2}\right)}{\pi} \ln \left[\sec \left(\frac{\pi a}{2 b}\right)\right]
$$

By inserting Eq. (26) into Eq. (24), we have

$$
k_{N, \text { dissinilar }}^{\text {arrap,approximate }}=k_{T, \text { dissimilar }}^{\text {array,approximate }}=\frac{G_{1}}{b\left(1+\kappa_{1}\right)} \frac{(1+\alpha)}{\left(1-\beta^{2}\right)\left(1+4 \varepsilon^{2}\right)} \pi\left\{\ln \left[\sec \left(\frac{\pi a}{2 b}\right)\right]\right\}^{-1} .
$$

The effect of elastic dissimilarity and crack density on the spring stiffness are explicitly expressed in terms of $a, \beta, \varepsilon$ and $a / b$ in both the exact (Eqs. (24) and (25)) and the approximate (Eq. (27)) forms. In the approximate form (Eq. (27)), however, the elastic mismatch and the crack density are no longer coupled as they are in the exact expression for $L(\varepsilon, a / b)$ in Eq. (25). It can be shown that both Eqs. (24) and (27) are symmetric with respect to indices 1 and 2 for material parameters based on the identity $\mathrm{G}_{1}(1+a) /\left(1+\kappa_{1}\right)=$ $G_{2}(1-a) /\left(1+\kappa_{2}\right)$.

The exact spring stiffness for non-interacting interfacial cracks in the dissimilar material case can be similarly obtained by using Eqs. (12), (22) and (23).

$$
k_{N, \text { dissimilar }}^{\text {non-interacting }}=k_{T, \text { dissimilar }}^{\text {non-interacting }}=\frac{8}{\pi} \frac{b}{a^{2}} \frac{G_{1}}{\left(1+\kappa_{1}\right)} \frac{(1+\alpha)}{\left(1-\beta^{2}\right)\left(1+4 \varepsilon^{2}\right)}
$$

It can be shown that as $a / b$ (crack density) approaches zero the approximate spring expression (27) for the array of cracks becomes Eq. (28), i.e. Eq. (28) is a limiting case of Eq. (27). Thus, the approximate equation (27) for interacting cracks, which neglects the 
effect of material dissimilarity on the crack interaction, becomes exact for the case of noninteracting cracks between dissimilar semispaces; this is a consistent result since the crack interaction in Eq. (28) is absent. One can show that Eq. (28) is identical to the result obtained by Pecorari and Kelly (2000), who have examined the spring stiffness for noninteracting cracks.

Eqs. (24) and (28), respectively, are reduced to the spring stiffness for the homogenous case by setting $a=\beta=\varepsilon=0$,

$$
k_{N, \text { homogenous }}^{\text {array }}=k_{T, \text { homogenous }}^{\text {array }}=\frac{G_{1}}{b\left(1+\kappa_{1}\right)} \pi\left[\ln \left\{\sec \left(\frac{\pi a}{2 b}\right)\right\}\right]^{-1} \text {, }
$$

and

$$
k_{N, h o m o g e n o u s}^{\text {non-interacting }}=k_{T, \text { homogenous }}^{\text {non-interacting }}=\frac{8}{\pi} \frac{b}{a^{2}} \frac{G_{1}}{\left(1+\kappa_{1}\right)},
$$

where the results obtained by Baik and Thompson (1984) are recovered with material 1 representing the homogeneous material.

\subsection{Effect of Elastic Dissimilarity of Semispaces on the Spring Stiffness}

When comparing the effective spring expressions for non-interacting cracks between homogenous material layers (Eq. (30)) and between dissimilar materials (Eq. (28)), we find that they differ by the factor $(1+\alpha) /\left[\left(1-\beta^{2}\right)\left(1+4 \varepsilon^{2}\right)\right]$. It is important to note that the same factor appears as the ratio of the approximate form of the effective spring stiffness for an array of cracks between dissimilar materials to that between homogenous material layers. Therefore, we define $M_{k}$ by the ratio of Eqs. (28) and (30) and that of Eqs. (27) and (29) as follows:

$$
M_{k}(\alpha, \beta)=\frac{k_{\text {dissimilar }}^{\text {non-interacting }}}{k_{\text {homogenous }}^{\text {non-interacting }}}=\frac{k_{\text {dissimilar }}^{\text {array,approximate }}}{k_{\text {homogenous }}^{\text {array }}}=\frac{(1+\alpha)}{\left(1-\beta^{2}\right)\left(1+4 \varepsilon^{2}\right)}
$$

where the subscript $N$ and $T$ are dropped since the normal and tangential spring stiffness are identical. Note that $M_{k}=1 / M_{W}$, where $M_{W}$ was introduced by Eq. (17); the relation is consistent with Eqs $(22,23)$. Since the Dundurs' parameters $a, \beta$ and the oscillation index, $\varepsilon$, all characterize the elastic dissimilarity between the semispaces, we can interpret $M_{k}$ as the elastic dissimilarity factor (representing the effect of elastic mismatch) for the effective spring stiffness for both non-interacting cracks and an array of cracks, and $M_{k}$ reduces to one for the homogeneous case $(\alpha=\beta=\varepsilon=0)$.

Figure 3 shows the elastic dissimilarity factor, $M_{k}$, as contour plots as a function of $\sigma$ and $\tau$ for all possible material combinations. Since $M_{k}$ is the inverse of $M_{W}$ which varies from 0.4209 to infinity (see Section 2.2), it is shown that $M_{W}$ varies from 0 to 2.376. Each cross symbol in Fig.3 represents a material combination provided by Suga et al. (1988) under the plane strain condition. Some commonly used material combinations are labeled with diamond shaped points. The $M_{k}$ contours are nearly parallel to the $\beta$ axis indicating that the a parameter is the dominant one in determining the value of $M_{k}$. At an a near $a \geq-1$, the contours are straight vertical lines. However, the curvature of contours is increasing with a reaching a maximum at $a=+1$. Note that $M_{k}$ is not symmetric with respect to indices 1 and 2 for the material parameters since transition to the homogeneous material is made by assuming that both materials are material 1 (Eq. (30)). 
Gorbatikh and Popova (2005) have shown that normal elastic compliances of noninteracting rectilinear, penny-shaped, elliptical, and annular non-interacting cracks between two dissimilar materials can be approximated by solutions for a homogeneous case through the common material parameter (Eq. (9) in Gorbatikh and Popova (2005)). It can be shown that their common material parameter, when expressed in terms of the Dundurs' parameters, is $(1+\alpha) /\left(1-\beta^{2}\right)$. One notes that it differs from $M_{k}$ equation (31) by the factor $1 /\left(1+4 \varepsilon^{2}\right)$,

which is close to one since the maximum value of $\varepsilon$ is $\frac{1}{2 \pi} \ln (3) \approx 0.1748$; and thus $M_{k}$ can be approximated by the material dissimilarity parameter introduced by Gorbatikh and Popova (2005). It is a significant result, since the elastic dissimilarity factor equation (31) is applicable to the case of crack interaction, and thus one can propose to extend the applicability of Eq. (31) from the 2D crack array to the interacting 3D plane cracks on an interface between dissimilar materials.

\subsection{The Effect of Crack Interaction on the Spring Stiffness}

It is well known (Kachanov (1994), Pecorari and Kelly (2000)) that interference between the crack tips for an array of periodic cracks in a homogenous material can be neglected if the crack density is less than 50\%. Exact equations (24) and (25) allow us to examine the validity of this non-interacting (independent) crack approximation in the context of effective interfacial spring stiffness between dissimilar materials.

In order to make a quantitative error estimate of crack interaction, we consider the ratio of effective spring stiffness for an array of cracks to that for non-interacting cracks

$$
I_{k}\left(\frac{a}{b}, \varepsilon\right)=\frac{k_{\text {dissimilar }}^{\text {array }}}{k_{\text {dissimilar }}^{\text {non-intering }}}=\frac{\pi}{8} \frac{a^{2}}{b^{2}} \frac{\left(1+4 \varepsilon^{2}\right)}{L\left(\frac{a}{b}, \varepsilon\right)},
$$

which depends on the integral representation of $L\left(\frac{a}{b}, \varepsilon\right)$ in Eq. (25). Given specific values of crack density $a / b$ and the oscillation index $\varepsilon, I_{k}$ describes the effect of crack interactions on the effective spring stiffness. Inserting Eq. (26) into Eq. (32), we obtain the approximate form of the interaction factor, which is identical to that for the homogeneous case ( $\varepsilon=0$ in Eq. (32))

$$
I_{k}\left(\frac{a}{b}, \varepsilon\right) \approx \frac{k_{\text {dissimilar }}^{\text {array,approximate }}}{k_{\text {dissimilar }}^{\text {non-interacting }}}=I_{k}^{\text {homogenous }}\left(\frac{a}{b}\right)=\frac{k_{\text {homogenous }}^{\text {array }}}{k_{\text {hom-interacting }}^{\text {nonous }}}=\frac{\pi^{2}}{8} \frac{a^{2}}{b^{2}}\left[\ln \left\{\sec \left(\frac{\pi a}{2 b}\right)\right\}\right]^{-1} .
$$

Based on Eqs. $(32,33)$, the effect of elastic mismatch on crack interactions can be examined for all possible values of crack density, $a / b$, ranging from one (noninteracting cracks or "independent" cracks) to zero (complete debond).

The exact crack interactions factor, equation (32), versus $a / b$ is shown with solid lines in Fig. 4 for the maximum value of $\varepsilon=\frac{1}{2 \pi} \ln (3) \approx 0.1748$ and $\varepsilon=0$ (the $\varepsilon=0$ case is identical to Eq. (33)). The interaction functions for both $\varepsilon=0$ (homogeneous case) and $\varepsilon=0.1748$ remain close to 0.9 at $a / b=0.5$ in the crack array, indicating that the crack interaction should be taken into account at crack density above $50 \%$ for both homogeneous and dissimilar material cases. In order to elucidate the effect of $\varepsilon$ on the interaction function, the ratios of the exact interaction function ( $\varepsilon=0.05,0.1$ and 0.1748$)$ and the interaction function for the homogeneous case $(\varepsilon=0)$ are also shown by dashed lines in Fig.4. It is interesting to note that the $I_{k}$ curve shown by the solid line for the homogeneous case $(\varepsilon=0)$ is slightly higher 
than the $I_{k}$ curve with $\varepsilon=0.1748$ over the wide $a / b$ range, and consequently the dashed $I_{k}$ ratio lines with $\varepsilon=0.05,0.1$ and 0.1748 are all below 1.0. This indicates that the interaction between cracks is slightly stronger for the dissimilar material case. With the maximum value of $\varepsilon=0.1748$, the interaction is highest. However the differences are not large; even for the extreme case of $95 \%$ of the crack density ( $a / b=0.95$ ), the $I_{k}$ ratio remains larger than $85 \%$ as shown by the dashed line for $\varepsilon=0.1748$. Considering that for most practical material pairs $\varepsilon$ is smaller than 0.05 , the effect of elastic dissimilarity on the crack interaction is negligible as is evident from Fig. 4, where the dashed $I_{k}$ ratio line with $\varepsilon=0.05$ remains at almost 1.0 for all values of crack density, $a / b$.

It is important to note that combination of Eqs. (24), (31) and (32) and of Eqs. (27), (31) and (33), respectively, leads to

$$
\begin{gathered}
k_{\text {dissimilar }}^{\text {array }}=I_{k} \times M_{k} \times k_{\text {homogenous }}^{\text {non-interacting }}, \\
k_{\text {dissimilar }}^{\text {array,approximate }}=I_{k}^{\text {homogenous }} \times M_{k} \times k_{\text {homogenous }}^{\text {non-interacting }} .
\end{gathered}
$$

As for the SERR, the effective spring stiffness for the array of cracks between dissimilar materials can be factorized in terms of crack interaction function $I_{k}$, elastic dissimilarity function $M_{k}$, and effective spring stiffness of non-interacting cracks for a homogenous material $k_{\text {homogenous }}^{\text {non-interacting }}$. For most realistic material pairs, the crack interaction in the dissimilar-material case can be approximated by that in a homogenous material.

It was noted (Kachanov and Laures, 1989) that the crack interaction in the homogeneous space is strongest for 2D strip cracks relative to 3D crack shapes. Thus our estimate provides an upper bound for the error when one replaces the spring interaction function for cracks between dissimilar semispaces by the same plane crack distribution in the homogeneous space.

\section{LOAD VALIDITY RANGE FOR ULTRASOUND APPLICATIONS}

\subsection{Evaluation of the Valid Loading Range Based on the Open Model}

All the derivations presented in Sections 2 and 3 are based on the assumption that crack faces are traction free (open) (Erdogan (1963), Williams (1959), Rice and Sih (1965)). The solution obtained under this condition, however, leads to oscillatory crack interpenetration zones at the crack tips, where the traction free condition, and thus solution, are not applicable. Comninou and co-workers (Comninou (1977), Comninou and Schmueser (1979), Schmueser and Comninou (1979)) have addressed the interpenetration zone problem by assuming a frictionless contact between the crack faces near the crack tips (the length of this contact zone is determined as a part of the problem solution). The replacement of the traction free boundary conditions by a unilateral contact zone at the crack tips leads to a nonlinear problem and a complicated iterative solution. However, if the interpenetration zone is much smaller than the crack length, the "small-scale contact conditions" (Rice, 1988) are satisfied, and the crack tip conditions are completely characterized by the open crack solution. Therefore, we would like to identify the range of loading for which smallscale contact conditions are satisfied and Eqs. (24) and (25) can be used.

Hills and Barber (1993) have provided excellent discussions of both the open formulation and the contact formulation. They have proposed a general expression for estimating the size of the interpenetration zone and have estimated the Comninou contact zone size from that of 
the interpenetration zone, which is larger than the contact zone. Graciani et al. (2007) have noticed that the Hills and Barber (1993) expression for the interpenetration zone is valid only for positive $\varepsilon$ and have modified it to be suitable for both positive and negative $\varepsilon$.

We employ the work by Graciani et al. (2007) to predict the interpenetration region for an array of cracks. Since materials 1 and 2 can be interchanged by allowing both positive and negative values of $\varepsilon$ without loss of generality, $\tau$ is applied in the positive $x$ direction. By combining Eq. (11) in Graciani et al. (2007) and Eqs. (1-3), we obtain the extent of the interpenetration zone $r_{i}$ from the right crack tip as

$\frac{r_{i}}{2 a}=\frac{\sin \left(\frac{\pi a}{b}\right)}{\left(\frac{\pi a}{b}\right)} \exp \left[\frac{1}{|\varepsilon|}\left\{\left(2 n-\frac{1}{2}\right) \pi-\tan ^{-1}\left(\frac{\tanh (\pi a \varepsilon / b)}{\tan (\pi a / 2 b)}\right) \operatorname{sgn} \varepsilon-\cot ^{-1}\left(\frac{\sigma}{\tau}\right) \operatorname{sgn} \varepsilon+\tan ^{-1}(2|\varepsilon|)\right\}\right]$.

Based on Eq. (36), it can be shown that the maximum interpenetration occurs at the right crack tip for negative $\varepsilon$. When the sign of $\varepsilon$ is switched to positive, the location of the maximum interpenetration zone is switched to the left crack tip. Therefore, for the purpose of evaluating the maximum interpenetration zone, it is sufficient to consider negative values of $\varepsilon$. When the interpenetration zone is limited to less than $1 \%$ of the full crack length (Rice, $1988)\left(r_{i} / 2 a<0.01\right)$, the valid loading range is estimated from Eq. (36) as follows:

$$
\left(\frac{\sigma}{\tau}\right) \geq \cot \left\{\frac{\pi}{2}-\tan ^{-1}\left(\frac{\tanh (\pi a \varepsilon / b)}{\tan (\pi a / 2 b)}\right)+\tan ^{-1}(2 \varepsilon)-\varepsilon \ln \left[\frac{0.01\left(\frac{\pi a}{b}\right)}{\sin \left(\frac{\pi a}{b}\right)}\right]\right\}
$$

The minimal values of stress ratio $\sigma / \tau$ required for negligible contact zone, as prescribed by Eq. (37), are given in Fig. 5 as contour plots. For most material combinations, the minimal stress ratio $\sigma / \tau$ varies from zero to one, indicating that the interpenetration zone is negligible as long as the tensile stress is larger than the shear stress. Only when $\varepsilon$ is zero (zero contour line, i.e. the homogeneous case), can far field pure shear be applied without encountering problems related to the interpenetration zone. Contour lines are approximately parallel to the $a / b$ axis (for $a / b<0.7$ ). Thus in this crack density range the minimal stress ratio required for negligible contact zone is mostly controlled by the parameter $\varepsilon$ and is independent of crack density (this is clear since the crack interaction is small).

\subsection{Ultrasound Applications}

Ultrasonic wave interaction with cracks is linear only if the crack surface displacements produced do not induce contact of crack surfaces. It is well known that ultrasonic waves reflect poorly from tightly closed cracks; and for closed interfacial cracks the ultrasonic wave reflection from a dissimilar material interface occurs in the same way as for the case without cracks. Thus experimental detection of interfacial cracks by ultrasonic waves is possible only when the cracks are slightly open, which is often the case due to loading history and plastic deformation. If under external or residual compressive stresses the crack is partially closed, the ultrasonic waves are perturbed only by its open part. If the elastodynamic displacements associated with the ultrasonic wave bridges a crack opening displacement, the wave interaction is nonlinear. The displacement amplitude of ultrasonic waves may vary by several orders of magnitude. Absolute amplitude measurements have been performed by optical interferometry methods and have been found to be in the subAngstrom to several nanometer range (Palmer et al., 1977 and Fick and Palmer, 2001).

Our model is based on the assumption that the crack surfaces are stress-free while the gap between the crack surfaces is zero in the absence of normal tension. To avoid crack surface 
interpenetration under ultrasonic shear load, one needs to apply a tension load to open the crack, thus mimicking realistic open crack conditions. Below we will estimate the degree of crack opening needed for the validity of the spring model (24) under an incident shear wave. The condition obtained will also satisfy the open crack model for the incident longitudinal wave.

By applying far field equivalent tensile stress, we can produce the required crack opening displacement and open the closed crack by the required amount. Therefore, even under pure shear load condition produced by an ultrasonic wave, the loading may be viewed as a combination of shear and equivalent tensile loads where the small-scale contact condition remains valid. This leads to a practical question as to what is the minimum initial crack opening required for the small-scale contact condition to be satisfied. To estimate this, we shift our attention from an array of cracks to independent (non-interacting) cracks (Hills et al., 1996). Since the crack opening is smallest when the cracks are not interacting (as can be shown in the homogeneous case based on the work by Koiter, 1959), the estimation we are seeking will provide us the most conservative estimation of minimal initial crack opening required. The midpoint of crack opening displacement, $\delta_{\max }$, of a single crack subjected to infinity equivalent tensile stress, $\sigma_{0}$, (Hills et al., 1996, Eq. (4.8)) can be expressed as

$$
\frac{\delta_{\max }}{a}=\frac{\sigma_{0}}{\tau} \frac{\tau}{2 G_{1}} A\left(\alpha, \beta, \kappa_{1}\right),
$$

where

$$
A\left(\alpha, \beta, \kappa_{1}\right)=\left(1+\kappa_{1}\right) \frac{\sqrt{1-\beta^{2}}}{(1+\alpha)} .
$$

In Eq. (38), we have used multiplication and division by an arbitrary magnitude of shear stress $\tau$, so the effect of shear and tensile combined loads on the maximum crack opening displacement can be examined. In Fig. 6, a contour plot of $A$ as a function of $\sigma$ and $\tau$ for $v_{1}=0.33$ is shown with several examples of material combinations labeled by circular points. Extreme material lines with $\kappa_{2}=1$ and $3\left(v_{2}=0.5\right.$ and 0$)$ are also shown in the figure. The contour lines are almost parallel to the $\beta$ axis, indicating that the effect of $\beta$ on the value of $A$ is small. Utilizing the weak dependence on $\beta$, we can write approximately

$$
A \approx \frac{\left(\kappa_{1}+1\right)}{(1+\alpha)}=\frac{G_{2}\left(\kappa_{1}+1\right)+G_{1}\left(\kappa_{2}+1\right)}{2 G_{2}} .
$$

As can be seen from Eqs. (39) and (40), $A$ is unbounded as a approaches $-1\left(G_{2} / G_{I}=0\right)$. The value of $A$ reaches approximately 100 for all values of $\beta$ when a is -0.98 . For all other values of $v_{1}$ varying from 0 to 0.5 , contour plots of $A$ are evaluated in a similar manner, and the maximum possible value of $A$ for most practical material combinations is estimated to be under 100. As we have already noted in the discussion of Figure 5, the minimum value of stress ratio $\sigma_{d} \tau$ which satisfies the condition of small scale contact zone for all material combination is approximately one. Assuming $\sigma / \tau=1$ and $\delta_{\max } / a \approx 10^{-5}(10 \mathrm{~nm}$ for a $1 \mathrm{~mm}$ crack length), Eq. (38) provides an estimation of strain, $\tau / 2 G_{1}$, to be of the order $10^{-7}$; this corresponds well to a reasonable level of strain in linear ultrasonics. Indeed, it will correspond to ultrasonic displacement of about an $\AA$ in the low $\mathrm{MHz}$ frequency range $(\lambda$ / $2 \sim 1 \mathrm{~mm})$ and in the range of directly measured $\left(10^{-7}-10^{-8}\right)$ ultrasonic strain (Alers and Fleury, 1964). This leads to the conclusion that the proposed effective spring stiffness is valid for small cracks as long as a preexisting midpoint crack opening is at least of order 
$10^{-5}$ of the half crack length. This crack opening also satisfies the open crack model requirement for an incident linear longitudinal ultrasonic wave.

\section{SUMMARY}

The longitudinal and transverse effective spring stiffnesses for a periodic array of collinear cracks located at the interface between two dissimilar materials are obtained based on the well-known open interface crack model. It is shown that they are identical to each other as in the case of elastically equal semispaces (homogeneous case); this result is not obvious due to coupling of normal and transverse tractions in the stress intensity factors. The spring stiffness is explicitly expressed in terms of the geometrical and the micromechanical properties of the interfacial cracks. When the crack density approaches zero, the obtained expression reduces to the effective spring stiffness for non-interacting cracks (Pecorari and Kelly, 2000). Similarly, the effective spring stiffness for a periodic array of collinear cracks in a homogeneous material (Angel and Achenbach (1985), Baik and Thompson (1984)) is recovered from the results obtained when the elastic dissimilarity of the semispaces vanishes.

It is demonstrated that the spring constant depends on the elastic dissimilarity of semispaces, the crack density and the crack interaction in the array. In general, the crack interaction weakly depends on material dissimilarity and, for most practical cases, this dependence can be replaced approximately by the interaction function of the crack array in the homogeneous space. It is also shown that the effective interfacial spring stiffness for non-interacting cracks can be used approximately for crack density below $50 \%$ as in the homogeneous case.

It is well known that in the open crack model the crack opening displacement exhibits an oscillatory crack interpenetration zone at the crack tip, thus violating the open crack assumption. To avoid the effect of the interpenetration zones on the effective spring stiffness the range of the tensile to transverse load ratios is obtained (as a function of the oscillation index, $\varepsilon$, and the crack density) such that the interpenetration zone is limited to $1 \%$ of the crack length. Since real cracks are often slightly open (due to prior loading history and plastic deformation), it is demonstrated for ultrasound applications that the results obtained can be readily used for most practical cases of small interfacial cracks as long as the midcrack opening normalized by the crack length is at least in the order of $10^{-5}$. (It is shown, that this is applicable for the level of shear strain below the order of $10^{-7}$ which is suitable for linear ultrasonic waves). Since the interfacial spring stiffness can be determined from ultrasound reflection and transmission analysis, the proposed expressions can be useful in estimating the percentage of disbond area between two dissimilar materials, which is directly related to the residual strength of the interface.

\section{Acknowledgments}

The authors acknowledge partial support of this research by NIHDCR C R21 DEO14719-0 grant; SIR also acknowledges partial support by NASA NNX08BA37A grant.

\section{Appendix}

Stress intensity factor, $K$, is provided in the handbook by Sih (1973) [Section 1.8.1-9] based on the work by Rice and Sih (1965) [Appendix 2] as follows.

$$
K=K_{I}+i K_{I I}=\sqrt{\pi} \cosh (\pi \varepsilon)\left(k_{1}+i k_{2}\right)
$$

where 


$$
k_{1}=\frac{2 \sqrt{b / \pi \sin (\pi a / b)}}{\cosh (\pi \varepsilon)}\left[\begin{array}{c}
\sigma\left\{\begin{array}{c}
\sin (\pi a / 2 b) \cosh (\pi a \varepsilon / b) \cos (\varepsilon \log [(2 b / \pi) \sin (\pi a / b)]) \\
+\cos (\pi a / 2 b) \sinh (\pi a \varepsilon / b) \sin (\varepsilon \log [(2 b / \pi) \sin (\pi a / b)])
\end{array}\right\} \\
+\tau\left\{\begin{array}{c}
\sin (\pi a / 2 b) \cosh (\pi a \varepsilon / b) \sin (\varepsilon \log [(2 b / \pi) \sin (\pi a / b)]) \\
-\cos (\pi a / 2 b) \sinh (\pi a \varepsilon / b) \cos (\varepsilon \log [(2 b / \pi) \sin (\pi a / b)])
\end{array}\right\}
\end{array}\right\}
$$

and

$$
k_{2}=\frac{2 \sqrt{b / \pi \sin (\pi a / b)}}{\cosh (\pi \varepsilon)}\left[\begin{array}{r}
\sigma\left\{\begin{array}{r}
-\sin (\pi a / 2 b) \cosh (\pi a \varepsilon / b) \sin (\varepsilon \log [(2 b / \pi) \sin (\pi a / b)]) \\
+\cos (\pi a / 2 b) \sinh (\pi a \varepsilon / b) \cos (\varepsilon \log [(2 b / \pi) \sin (\pi a / b)])
\end{array}\right\} \\
+\tau\left\{\begin{array}{l}
\sin (\pi a / 2 b) \cosh (\pi a \varepsilon / b) \cos (\varepsilon \log [(2 b / \pi) \sin (\pi a / b)]) \\
+\cos (\pi a / 2 b) \sinh (\pi a \varepsilon / b) \sin (\varepsilon \log [(2 b / \pi) \sin (\pi a / b)])
\end{array}\right\}
\end{array}\right]
$$

where $\varepsilon$ is given by Eq. (2) and Eq. (3) can be expressed as

$$
\begin{aligned}
& \beta=\frac{1}{2} \frac{G_{2}\left(1+v_{2}\right)\left(1-v_{1}\right)-G_{1}\left(1+v_{1}\right)\left(1-v_{2}\right)}{G_{2}\left(1+v_{2}\right)+G_{1}\left(1+v_{1}\right)} \text { for plane stress, } \\
& \beta=\frac{1}{2} \frac{G_{2}\left(1-2 v_{1}\right)-G_{1}\left(1-2 v_{2}\right)}{G_{2}\left(1-v_{1}\right)+G_{1}\left(1-v_{2}\right)} \text { for plane strain. }
\end{aligned}
$$

\section{REFERENCES}

Angel YC, Achenbach JD. Reflection and transmission of elastic waves by a periodic array of cracks. Journal of Applied Mechanics-Transactions of the ASME. 1985; 52(1):33-41.

Alers GA, Fleury PA. Strain amplitude of Mc/sec ultrasonic waves in solids. The Journal of the Acoustical Society of America. 1964; 36(7):1297-1304.

Baik J, Thompson RB. Ultrasonic scattering from imperfect interfaces: a quasi-static model. Journal of Nondestructive Evaluation. 1984; 4(3/4):177-196.

Baltazar A, Wang L, Xie B, Rokhlin SI. Inverse ultrasonic determination of imperfect interfaces and bulk properties of a layer between two solids. Journal of the Acoustical Society of America. 2003; 114:1424-1434. [PubMed: 14514195]

Comninou M, Schmueser D. The interface crack in a combined tension-compression and shear field. Journal of Applied Mechanics-Transactions of the ASME. 1979; 46(2):345-348.

Comninou M. The interface Crack. Journal of Applied Mechanics-Transactions of the ASME. 1977; 44(4):631-636.

Dundurs J. Effect of elastic constants on stress in a composite under plane deformation. Journal of Composite Materials. 1967; 1:310-322.

Dundurs J, Bogy DB. Edge-bonded dissimilar orthogonal elastic wedges under normal and shear loading. Journal of Applied Mechanics. 1969; 36(3):650-652.

England AH. A crack between dissimilar media. Journal of Applied Mechanics. 1965; 32:400-402.

Erdogan F. Stress distribution in a nonhomogenous elastic plane with cracks. Journal of Applied Mechanics. 1963; 30:232-236.

Erdogan F. Stress distribution in bonded dissimilar materials with cracks. Journal of Applied Mechanics. 1965; 32(2):403-410.

Fick SE, Palmer CH. Precision ultrasonic wave measurements with simple equipment. Journal of Research of the National Institute of Standards and Technology. 2001; 106:833-841.

Gorbatikh L, Popova M. Estimation of elastic compliances of planar interfacial cracks from homogeneous solutions. Applied Physics Letters. 2005; 86(3) 031905.

Graciani E, Mantic V, Paris F. On the estimation of the first interpenetration point in the open model of interface cracks. International Journal of Fracture. 2007; 143:287-290.

Hills, DA.; Kelly, PA.; Dai, DN.; Korsunsky, AM. Solution of crack problems: The distributed dislocation technique. Kluwer Academic Publishers; 1996. 
Hills DA, Barber JR. Interface cracks. International Journal of Mechanical Sciences. 1993; 35(1):2737.

Hutchinson JW, Mear ME, Rice JR. Crack paralleling an interface between dissimilar materials. Journal of Applied Mechanics-Transactions of the ASME. 1987; 54:828-832.

Hutchinson JW, Suo Z. Mixed mode cracking in layered materials. Advances in Applied Mechanics. 1992; 29:63-191.

Kachanov M, Laures JP. Three-dimensional problems of strongly interacting arbitrarily located pennyshaped cracks. 1989; 41:289-313.

Kachanov M. Elastic solids with many cracks and related problems. Advances in Applied Mechanics. 1994; 30:259-445.

Koiter WT. An infinite row of collinear cracks in an infinite elastic sheet. Archive of Applied Mechanics. 1959; 28(1):168-172.

Lavrentyev AI, Rokhlin SI. Models for ultrasonic characterization of environmental degradation of interfaces in adhesive joints. Journal of Applied Physics. 1994; 76(8):4643-4650.

Leiderman R, Barbone PE, Braga AMB. Reconstructing the adhesion stiffness distribution in a laminated elastic plate: Exact and approximate inverse scattering solutions. Journal of the Acoustical Society of America. 2007; 122(4):1906-1916. [PubMed: 17902827]

Margetan FJ, Thompson RB, Gray TA. Interfacial spring model for ultrasonic interactions with imperfect interfaces: theory of oblique incidence and application to diffusion-bonded butt joints. Journal of Nondestructive Evaluation. 1988; 7(3/4):131-152.

Miller RA. Current status of thermal barrier coatings: an overview. Surface Coating Technology. 1987; 30:1-11.

Nemat-Nasser S, Yu N, Hori M. Solids with periodically distributed cracks. International Journal of Solids and Structures. 1993; 30(15):2071-2095.

Palmer DD, Rehbein DK, Smith JF, Buck O. Nondestructive Characterization of mechanical strength of diffusion bonds, II. Application of a quasi-static spring model. Journal of Nondestructive Evaluation. 1988; 7(3/4):167-174.

Palmer CH, Claus RO, Fick SE. Ultrasonic wave measurement by differential interferometry. Applied Optics. 1977; 16:1849-1856. [PubMed: 20168821]

Pecorari C, Kelly PA. The quasi-static approximation for a cracked interface between a layer and a substrate. Journal of the Acoustical Society of America. 2000; 107(5):2454-2461. [PubMed: 10830368]

Rice JR, Sih GC. Plane problems of cracks in dissimilar media. Journal of Applied Mechanics. 1965; 32(2):418-423.

Rice JR. Elastic fracture mechanics concepts for interfacial cracks. Journal of Applied MechanicsTransactions of the ASME. 1988; 55(1):98-103.

Rokhlin SI, Xie B, Baltazar A. Quantitative ultrasonic characterization of environmental degradation of adhesive bonds. Journal of Adhesion Science and Technology. 2004; 18(3):327-359.

Schmueser D, Comninou M. Periodic array of interface cracks and their interaction. International Journal of Solids and Structures. 1979; 15(12):927-934.

Sih, GC. Handbook of stress-intensity factors for researchers and engineers. Institute of Fracture and Solid Mechanics, Lehigh University; 1973.

Suga T, Elssner G, Schmauder S. Composite Parameters and Mechanical Compatibility of Material Joints. Journal of Composite Materials. 1988; 22:917-934.

Tada, H.; Paris, PC.; Irwin, GR. The stress analysis of cracks handbook. New York, NY: ASME Press; 2000.

Thompson RB, Thompson DO. Past experiences in the development of tests for adhesive bond strength. Journal of Adhesion Science and Technology. 1991; 5(8):583-599.

Wang Y, Katsube N, Seghi RR, Rokhlin SI. Statistical failure analysis of adhesive resin cement bonded dental ceramics. Engineering Fracture Mechanics. 2007; 74:1838-1856. [PubMed: 18670583]

Wang H, Qian ML, Liu W. Laser ultrasonic characterization of adhesive bonds between epoxy coating and aluminum substrate. Ultrasonics. 2006; 44:E1349-E1353. [PubMed: 16782159] 
Wang W, Rokhlin SI. Evaluation of interfacial properties in adhesive joints of aluminum-alloys using angle-beam ultrasonic spectroscopy. Journal of Adhesion Science and Technology. 1991; 5(8): 647-666.

Williams ML. The stress around a fault or crack in dissimilar media. Bulletin of the Seismological Society of America. 1959; 49:199-204. 
(a)

Periodic array of interfacial cracks between dissimilar materials two

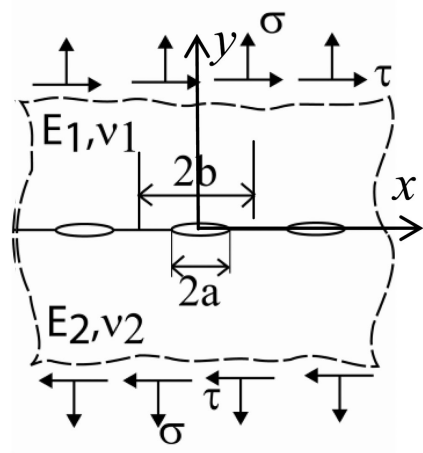

(b)

Periodic array cracks replaced by distributed springs
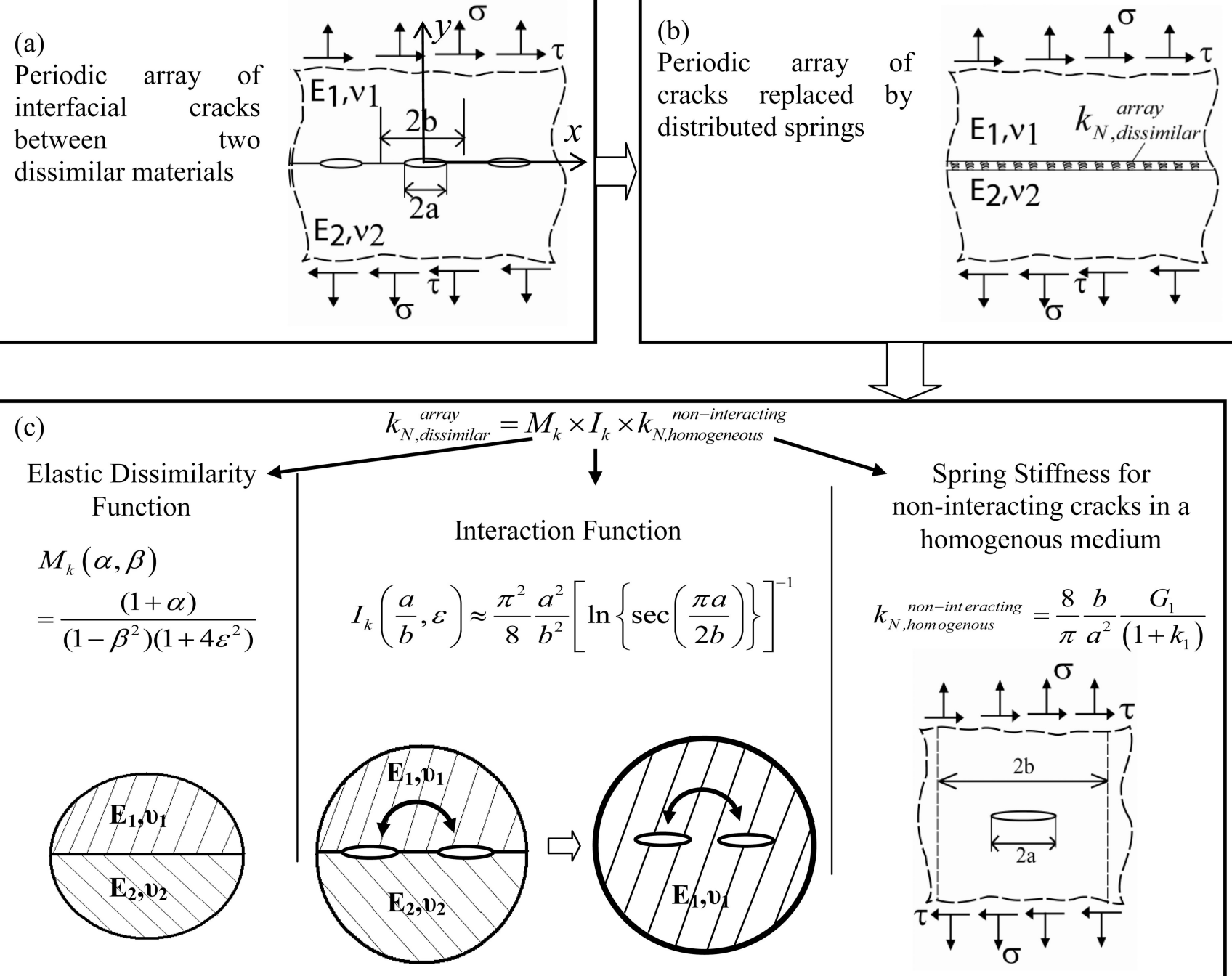

Figure 1.

Effective spring stiffness for a planar periodic array of collinear cracks between two dissimilar isotropic materials 


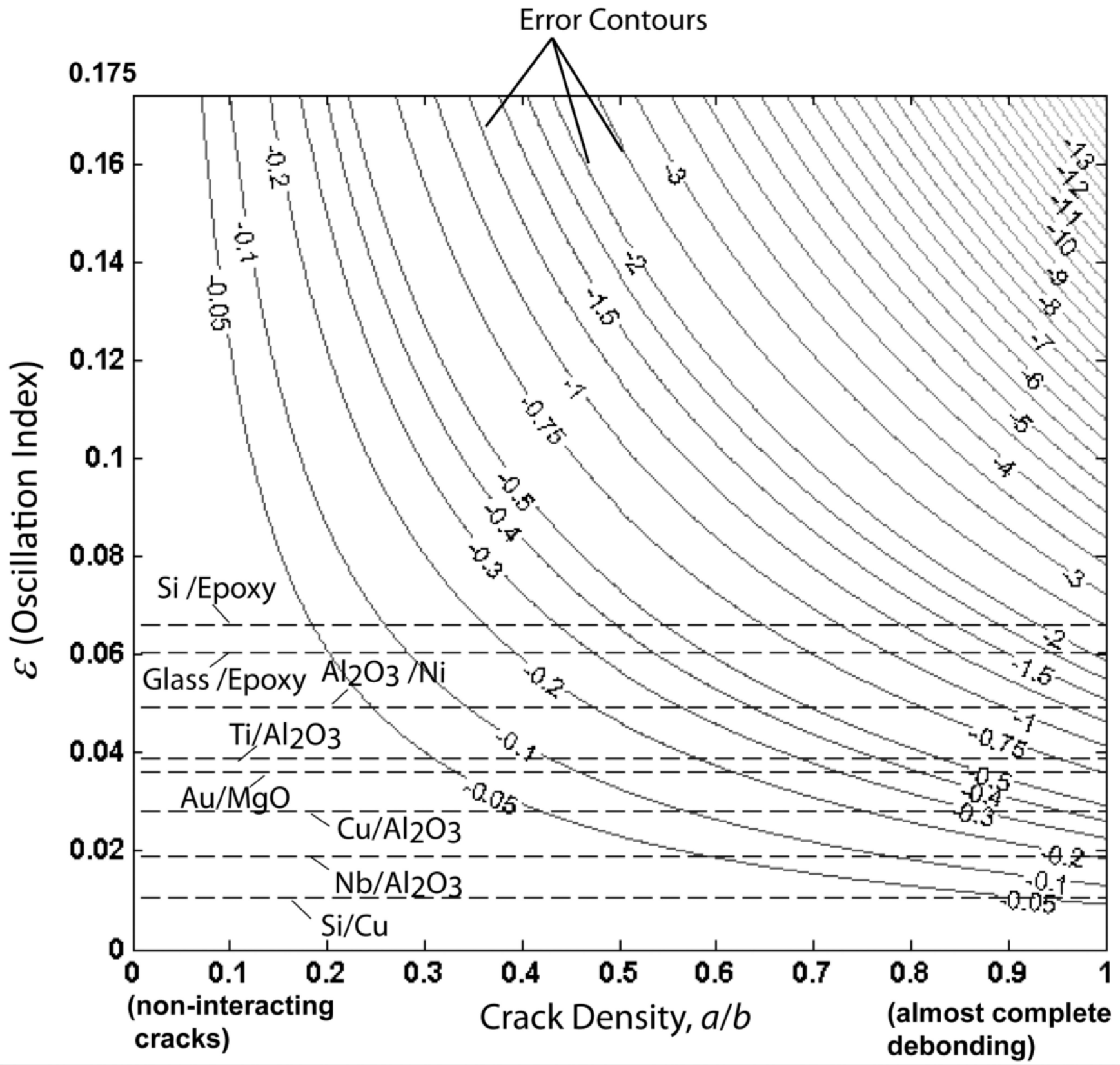

Figure 2.

Contour plot of percent error of approximate interaction function $I_{W}(a / b)$ relative to the exact interaction function $I_{W}(a / b, \varepsilon)$ with some material combination examples (Example combinations are taken from Hutchinson et al. 1987.) 




Figure 3.

Contour plot of elastic dissimilarity function for effective spring stiffness. Each cross symbol (x) represents a material combination provided by Suga et al. (1988) for plane strain. Some material combinations are indicated by circle and labeled. 


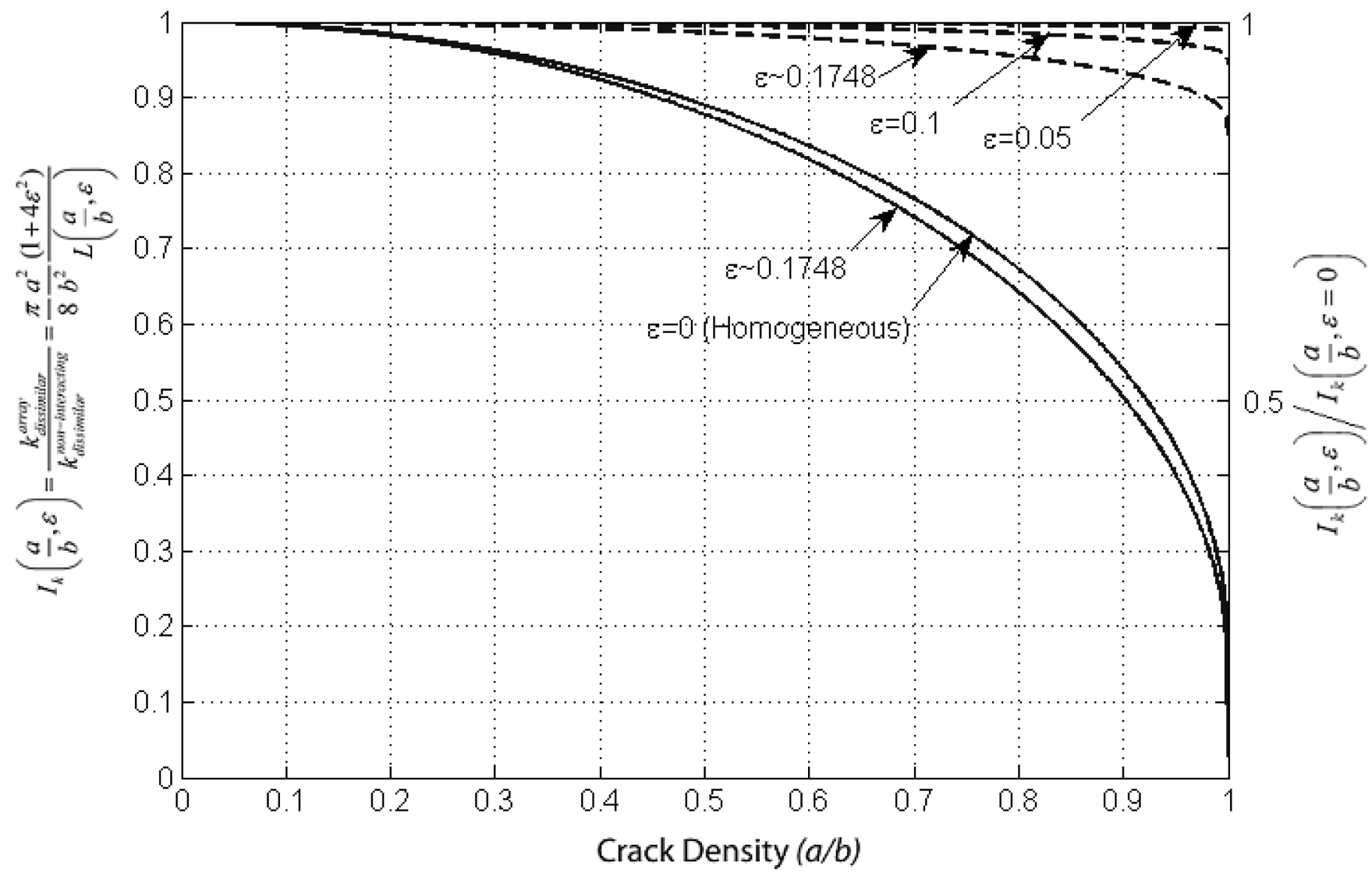

Figure 4.

Exact interaction function for effective spring stiffness with $\varepsilon \approx 0.1748$ and $\varepsilon=0$ (solid lines) and ratios of exact interaction function ( $\varepsilon \approx 0.1748, \varepsilon=0.1$ and $\varepsilon=0.05)$ with the interaction function to homogenous case $\varepsilon \approx 0$ (dashed lines). 


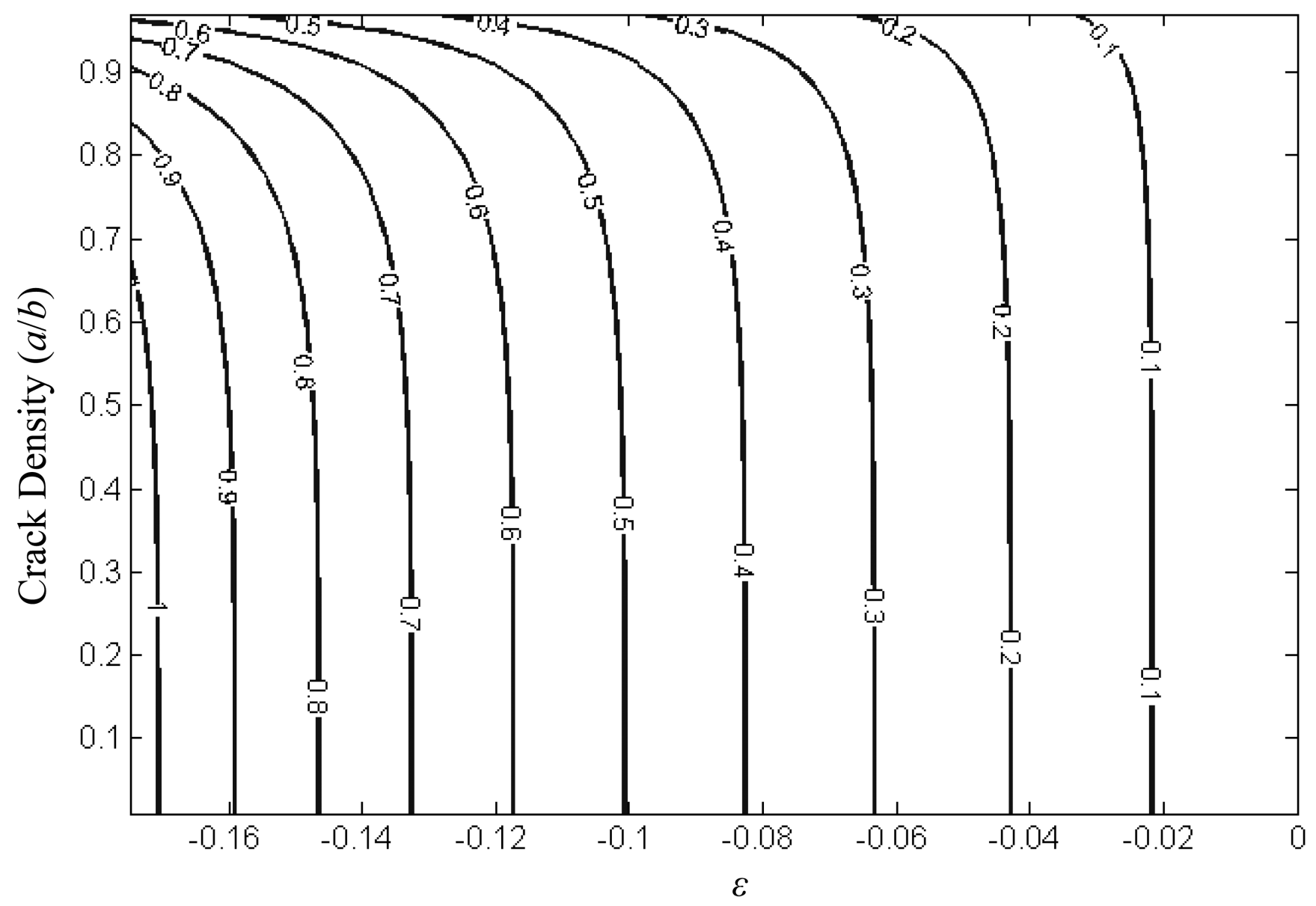

Figure 5.

Contour plots of the minimal stress ratio $\sigma / \tau$ required for negligible contact zone as function of $\varepsilon$ and crack density (The interpenetration zone size $r_{i}$ is below $1 \%$ of crack length and it occurs at the right crack tip for $\varepsilon<0$.) 


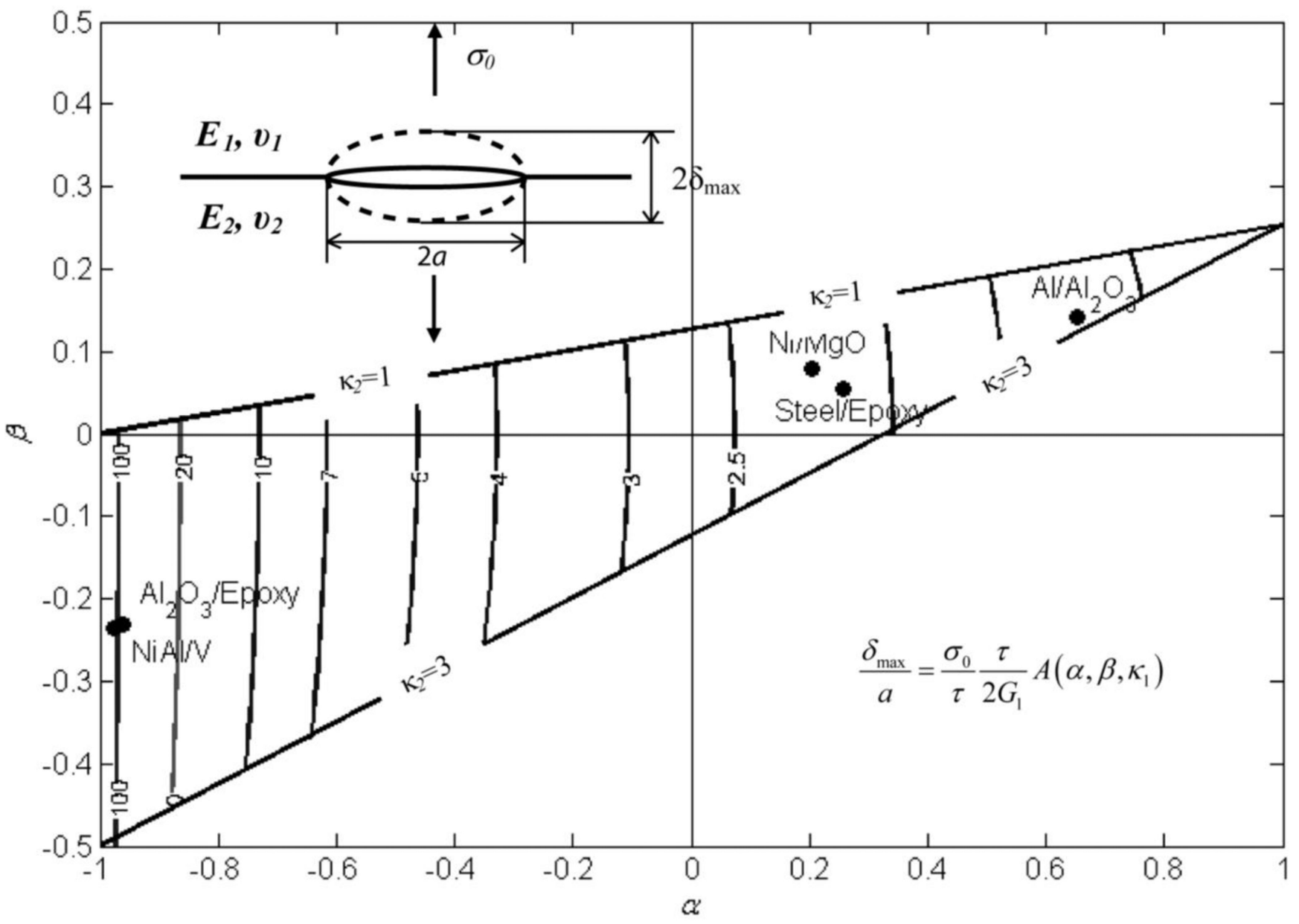

Figure 6.

Contour plot of parameter $A\left(\alpha, \beta, \kappa_{1}\right)$ for $v_{1}=0.33$. Some material combinations with $v_{1}=0.33$ are indicated by circle and labeled. 